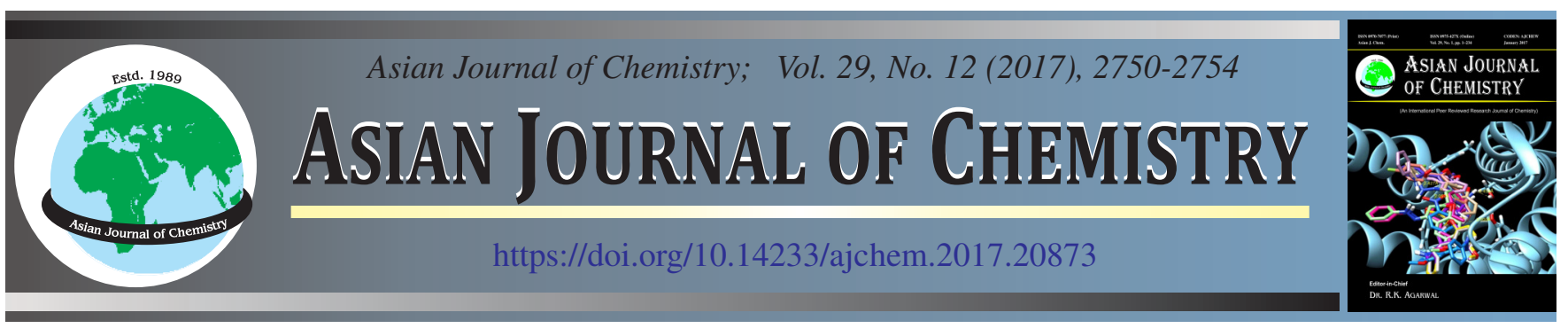

\title{
Microwave Assisted Gould-Jacob Reaction in the Synthesis of Furopyrimidopyrimidines and Their Transformation via Ring Opening to Aminoesters
}

\author{
NiRmal M. Shah, Vivek C. Ramani and Rina D. ShaH*
}

Department of Chemistry, M.G. Science Institute, Navrangpura, Ahmedabad-380 009. India

*Corresponding author: E-mail: drrdshah@yahoo.co.in

\begin{abstract}
Gould-Jacob reaction is well-known for the construction of fused pyrimidopyrimidines from pyrimidineamines and diethyl ethoxymethylenemalonate (EMME) or acyl malonic ester. Ethyl 4-oxo-8,9-disubstitutedfuro[3,2-e]pyrimidine-3-carboxylates (3) has been carried out by classical heating from furo[2,3-d]pyrimidine-4-amines (1) and diethyl ethoxymethylenemalonate through the formation of uncyclized diethyl $\mathrm{N}$-[5,6-substituted-7H-[2,3-d]pyrimidin-4-yl]aminomethylenemalonates (2) as an intermediate. The identical compound 3 have also been synthesized via rapid solvent free microwave irradiation method. A comparison of classical method versus solvent-free microwave irradiation method has been studied. The reaction of compound $\mathbf{3}$ has also been explored with alcoholic $\mathrm{NaOH}$ and hydrazine hydrate to synthesize corresponding acid $\mathbf{4}$ and acid hydrazide derivatives $\mathbf{5}$. Reaction of compound $\mathbf{3}$ with hydrazine hydrate failed to yield acid hydrazide, but they underwent unexpected ring opening and transformed to respective aminoester 6.
\end{abstract}

Keywords: Gould-Jacob reaction, Furopyrimidopyrimidines, Ethoxymethylene malonate, Alkaline hydrolysis, Aminoesters.

\section{INTRODUCTION}

Synergism of microwave acceleration with solvent-free reaction conditions [1-3] is always been interesting to study as it provides an environmentally benign system. Gould-Jacob reaction for the synthesis of quinoline derivatives has been attempted using diethyl ethoxymethylene malonate (EMME) as synthon [4]. Diethyl ethoxymethylene malonate, a versatile reagent is widely used in push-pull alkane [5], 1,4-addition elimination [6], 1,4-addition [7], [3+2] cyclo-additions [8], DielsAlder reactions [9] and extensively reviewed as Michael reagent [10]. Synthesis of 1,8-naphthyridines, $2 H$-pyrido[1,2-a]pyrimidin4-ones, pyrazolinones, pyrons, xanthyrones, guanidine derivatives, 1,2,4-triazoles, 3-oxo-1,2,6-thiadiazines, 8-oxoimidazo[1,2-a]pyrimidines, 3H-pyrrolo[1,2-a]indol-3-one derivatives, $1 \mathrm{H}-1,4-$ benzodiazepines, pyrido[3,2-e]pyrimido-[1,2-c]pyrimidines have been reported employing EMME [11,12]. Microwave assisted solvent-free synthesis of thieno[3,2-e]-pyrimido[1,2-c]pyrimidines and pyrrolo[3,2-e]pyrimido[1,2-c]-pyrimidines have also been attempted $[13,14]$. Microwave assisted synthesis have become attractive during last two decades as it is rapid, cleaner, yield increasing and purity enhancing [13-18], sometimes allows solvent-free conditions $[1,2,13,14]$. It has always been tempting to attend solvent free organic synthesis as it resolves environ-mental issues [19]. Pyridopyrimidine obtained from EMME also identified as anticancer and antimicrobial agents
[20]. So far synthesis of furopyrimidopyrimidines have not been reported from EMME. Therefore in continuation of our interest [12-14,21-34] in the synthesis of angular trihetero-cycles, we herein report the synthesis of furo[3,2-e]pyrimido[1,2c]pyrimidines (2) by well-known Gould-Jacob type of reaction from furo[2,3- $d$ ]-pyrimidine-4-amines (1). A comparative study of conventional as well as microwave methodologies has also been under taken for the synthesis of furopyrimidopyrimidines (3). The reaction of compound $\mathbf{3}$ has been carried with alcoholic $\mathrm{NaOH}$ to form respective acids 4 (Scheme-I).

Reaction of $\mathbf{3 c}$ has also been explored with hydrazine hydrate that underwent ring cleavage to form aminoester $\mathbf{6 c}$ rather giving acid hydrazide 5c (Scheme-II).

\section{EXPERIMENTAL}

Melting points were determined by electro thermal method in open capillary tube and are uncorrected. The IR spectra were recorded in $\mathrm{cm}^{-1}$ for $\mathrm{KBr}$ pellets on Buck scientific spectrophotometer. The ${ }^{1} \mathrm{H}$ NMR spectra were recorded on Varian $300 \mathrm{MHz}$ or Bruker $400 \mathrm{MHz}$ spectrophotometer in DMSO$d_{6}$ or $\mathrm{CDCl}_{3}$ using TMS as internal standard and the chemical shifts are expressed in ppm. MS spectra were recorded on LKB 9000 mass spectrophotometer. Microwave irradiation was carried out in CEM Discover microwave, Model No 90801 (2455 MHz, $700 \mathrm{~W}$ ). The purity of newly synthesized compounds was routinely 


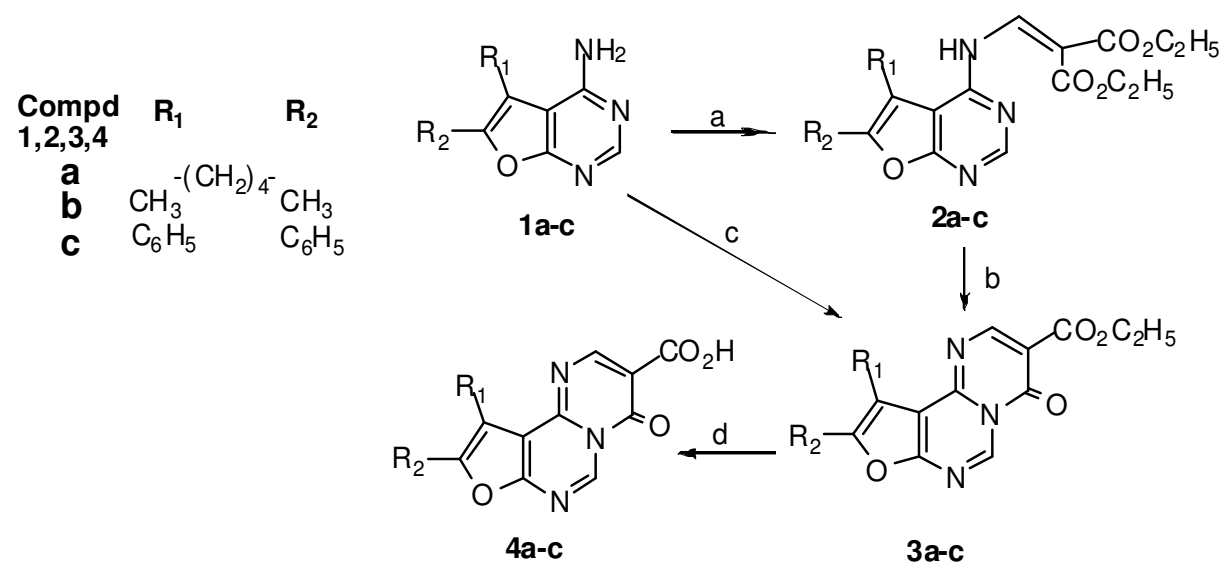

[a] EMME, $130-40^{\circ} \mathrm{C}, 3.5-4 \mathrm{~h}$, [b] DPO, $250^{\circ} \mathrm{C}, 1.5-2.0 \mathrm{~h}$, [c] MW, 8-9 $\min$ [d] $2 \mathrm{~N} \mathrm{NaOH}$, ethanol, reflux, $2 \mathrm{~h}$ Scheme-I

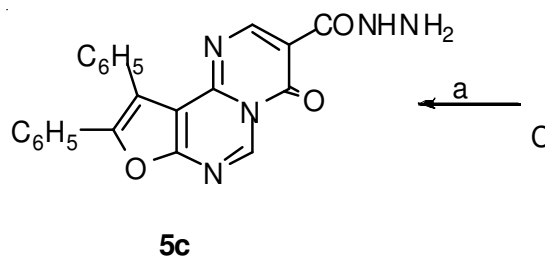

$[a] \mathrm{NH}_{2} \mathrm{NH}_{2}, \mathrm{EtOH}$, reflux, $1 \mathrm{~h}$

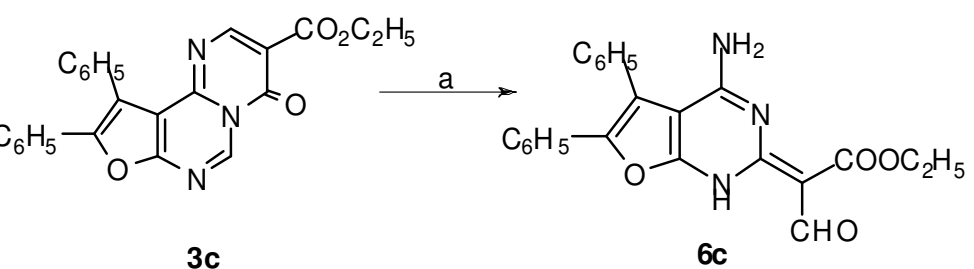

$3 c$

$6 c$

Scheme-II

checked by TLC using silica gel G and spots were exposed in iodine vapour.

General procedure for the synthesis of ethyl 4-oxo-8,9disubstituted furo[3,2-e]-pyrimido[1,2-c]pyrimidine-3carboxylates (3a-e)

\section{Method-A: Two step conventional method}

Step-1: Synthesis of diethyl N-[5,7-substituted furo[2,3d]pyrimidin-4-yl]amino-methylenemalonates (2a-c): A mixture of 5,6-disubstituted furo[2,3- $d$ ] pyrimidine-4-amines [35] (1) (0.005 mol) and EMME (1.08 g $0.005 \mathrm{~mol})$ was heated at $130-140{ }^{\circ} \mathrm{C}$ for 3.5 to $4 \mathrm{~h}$. It was allowed to cool, treated with chilled methanol and the obtained solid was filtered, washed with chilled methanol, dried and recrystallized.

Diethyl N-[cyclohexa[5,6]furo[2,3- $d$ ] pyrimidin-4-yl]aminomethylenemalonate (2a): Yield: $77 \%$, m.p.: 149-50 ${ }^{\circ} \mathrm{C}$, IR $\left(\mathrm{KBr}, \mathrm{v}_{\max }, \mathrm{cm}^{-1}\right): 3255(\mathrm{NH}), 1697,1676(\mathrm{C}=\mathrm{O}), 1602$, $1502(\mathrm{C}=\mathrm{C}, \mathrm{C}=\mathrm{N}) .{ }^{1} \mathrm{H}$ NMR $\left(\mathrm{CDCl}_{3}\right): \delta 11.59-11.63(\mathrm{~d}, J=$ $11.88 \mathrm{~Hz}, 1 \mathrm{H}, \mathrm{NH}), \delta 9.34-9.36(\mathrm{~d}, J=12.03 \mathrm{~Hz}, 1 \mathrm{H}$, vinyl-H), $\delta 8.63(\mathrm{~s}, 1 \mathrm{H}, \mathrm{ArH}$ at C2), 4.26-4.39 (qx2, $J=5.59 \mathrm{~Hz}, 4 \mathrm{H}$, $\mathrm{CH}_{2}$-ester), 3.11-3.14 (t, $J=5.91 \mathrm{~Hz}, 2 \mathrm{H}, \mathrm{CH}_{2} \mathrm{CH}_{2} \mathrm{CH}_{2} \mathrm{CH}_{2}$ ), 2.85-2.89 (t, $J=5.87 \mathrm{~Hz}, 2 \mathrm{H}, \underline{\mathrm{CH}}_{2} \mathrm{CH}_{2} \mathrm{CH}_{2} \mathrm{CH}_{2}$ ), 1.79-2.06 $\left(\mathrm{m}, J=4.06 \mathrm{~Hz}, 4 \mathrm{H}, \mathrm{CH}_{2} \mathrm{CH}_{2} \mathrm{CH}_{2} \mathrm{CH}_{2}\right) 1.35-1.42$ (tx2, $J=$ $6.86 \mathrm{~Hz}, 6 \mathrm{H}, \mathrm{CH}_{3}$-ester). MS: $359\left(\mathrm{M}^{+}\right)$, Anal. calcd. (\%) for $\mathrm{C}_{18} \mathrm{H}_{21} \mathrm{~N}_{3} \mathrm{O}_{5}$ : C, 60.16; H, 11.69; N, 22.26; Found: C, 60.24; $\mathrm{H}, 11.87$; N, 22.35.

Diethyl N-[5,6-dimethylfuro[2,3- $d$ ]pyrimidin-4-yl]aminomethylenemalonate (2b): Yield: $81 \%$, m.p.: $139-40{ }^{\circ} \mathrm{C}$, IR $\left(\mathrm{KBr}, v_{\max }, \mathrm{cm}^{-1}\right): 3254(\mathrm{NH}), 1696,1670(\mathrm{C}=\mathrm{O}), 1601,1500$ $(\mathrm{C}=\mathrm{C}, \mathrm{C}=\mathrm{N}) .{ }^{1} \mathrm{H} \mathrm{NMR}\left(\mathrm{CDCl}_{3}\right): \delta 10.5-10.54(\mathrm{~d}, J=11.89 \mathrm{~Hz}$, $1 \mathrm{H}, \mathrm{NH}), \delta 9.3-9.32(\mathrm{~d}, J=12.04 \mathrm{~Hz}, 1 \mathrm{H}$, vinyl-H), $\delta 8.65$ (s, $1 \mathrm{H}, \mathrm{ArH}$ at C2); 4.03-4.29 (qx2, $J=6.05 \mathrm{~Hz}, 4 \mathrm{H}, \mathrm{CH}_{2}$-ester), $2.4\left(\mathrm{~s}, 3 \mathrm{H}, \mathrm{CH}_{3}\right), 1.98\left(\mathrm{~s}, 3 \mathrm{H}, \mathrm{CH}_{3}\right), 1.22-1.35(\mathrm{tx} 2, J=7.26$ $\mathrm{Hz}, 6 \mathrm{H}, \mathrm{CH}_{3}$-ester). MS: $303\left(\mathrm{M}^{+}\right)$, Anal. calcd. (\%) for $\mathrm{C}_{14} \mathrm{H}_{13} \mathrm{~N}_{3} \mathrm{O}_{5}$ : C, 55.45; H, 4.32; N, 13.86; Found: C, 55.40; H, 4.23; N, 13.56 .

Diethyl N-[5,6-diphenylfuro[2,3-d]pyrimidin-4-yl]aminomethylenemalonate (2c): Yield: $78 \%$, m.p.: $121^{\circ} \mathrm{C}$, IR $\left(\mathrm{KBr}, \mathrm{v}_{\max }, \mathrm{cm}^{-1}\right): 3258(\mathrm{NH}), 1700,1674(\mathrm{C}=\mathrm{O}), 1603,1505$ $(\mathrm{C}=\mathrm{C}, \mathrm{C}=\mathrm{N}) .{ }^{1} \mathrm{H}$ NMR $\left(\mathrm{CDCl}_{3}\right): \delta 10.55-10.59(\mathrm{~d}, J=11.97 \mathrm{~Hz}$, $1 \mathrm{H}, \mathrm{NH}), \delta 9.22-9.27(\mathrm{~d}, J=12.21 \mathrm{~Hz}, 1 \mathrm{H}$, vinyl-H), $\delta 8.78$ (s, $1 \mathrm{H}, \mathrm{ArH}$ at C2); $\delta$ 7-7.36 (m, 10H, ArH), 4.02-4.29 (qx2, $J$ $=6.57 \mathrm{~Hz}, 4 \mathrm{H}, \mathrm{CH}_{2}$-ester $), 1.23-1.35(\mathrm{tx} 2, J=7.29 \mathrm{~Hz}, 6 \mathrm{H}$, $\mathrm{CH}_{3}$-ester). MS: $457\left(\mathrm{M}^{+}\right)$, Anal. calcd. (\%) for $\mathrm{C}_{26} \mathrm{H}_{23} \mathrm{~N}_{3} \mathrm{O}_{5}$ : C 68.26, H 5.7, N 9.19; Found: C, 68.16; H, 5.51; N, 9.01.

Step 2: Synthesis of ethyl 4-oxo-8,9-disubstitutedfuro[3,2-e]pyrimido[1,2-c]pyrimidine-3-carboxylates (3a-c): Diethyl N-[5,6-disubstituted 7H-furo[2,3- $d$ ]pyrimidin-4-yl]aminomethylene malonate (3) (1 g) was dissolved in boiling diphenyl oxide (DPO) $(5 \mathrm{~mL})$ and heated at $250{ }^{\circ} \mathrm{C}$ for $1.5-2 \mathrm{~h}$. The excess of solvent distilled in vacuo and methanol $(15 \mathrm{~mL})$ was added to the cold reaction mixture, the solid obtained was collected by filtration and crystallized from DMF:ethanol (6:4 v/v).

\section{Method B}

Ethyl 4-oxo-8,9-substitutedfuro[3,2-e]pyrimido[1,2-c]pyrimidine-3-carboxylates (3a-c): A neat equimolar mixture of 5,6-disubstituted furo[2,3- $d$ ]pyrimidine-4-amines (1) (0.005 mol) and EMME (1.08 g $0.005 \mathrm{~mol})$ was taken in a Pyrex tube and subjected to microwave irradiation in a microwave oven at an output of 700 watts at $150{ }^{\circ} \mathrm{C}$ for specified time mentioned in (Table-1). Progress of reaction was monitored through TLC at an interval of $45 \mathrm{~s}$. On completion, the reaction mixture was allowed to cool at room temperature and the solid obtained was crystallized from DMF:EtOH (6:4 v/v). 
Ethyl 4-oxocyclohexa[8,9]furo[3,2-e]pyrimido[1,2-c]pyrimidine-3-carboxylate (3a): m.p.: $214^{\circ} \mathrm{C}$, IR (KBr, $v_{\max }$, $\left.\mathrm{cm}^{-1}\right): 1720,1685(\mathrm{C}=\mathrm{O}), 1603,1506(\mathrm{C}=\mathrm{C}, \mathrm{C}=\mathrm{N}) .{ }^{1} \mathrm{H}$ NMR $\left(\mathrm{CDCl}_{3}\right): \delta 9.1$ (s, 1H, Ar-H at C6), $\delta 7.25(\mathrm{~s}, 1 \mathrm{H}, \mathrm{Ar}-\mathrm{H}$ at $\mathrm{C} 2)$, 4.4-4.46 (q, $J=6.95 \mathrm{~Hz}, 4 \mathrm{H}, \mathrm{CH}_{2}$-ester), 3.25-3.27 (t, $J=$ $\left.3.71 \mathrm{~Hz}, 2 \mathrm{H}, \mathrm{CH}_{2} \mathrm{CH}_{2} \mathrm{CH}_{2} \underline{\mathrm{CH}}_{2}\right), 2.92-2.95(\mathrm{t}, J=4.6 \mathrm{~Hz}$, $\left.2 \mathrm{H}, \mathrm{CH}_{2} \mathrm{CH}_{2} \mathrm{CH}_{2} \mathrm{CH}_{2}\right), 1.91-1.99(\mathrm{~m}, J=6.56 \mathrm{~Hz}, 4 \mathrm{H}$, $\mathrm{CH}_{2} \underline{\mathrm{CH}}_{2} \underline{\mathrm{CH}}_{2} \mathrm{CH}_{2}$ ), 1.4-1.45 (t, $J=6.06 \mathrm{~Hz}, 3 \mathrm{H}, \mathrm{CH}_{3}$-ester). MS: $313\left(\mathrm{M}^{+}\right)$, Anal. calcd. (\%) for $\mathrm{C}_{16} \mathrm{H}_{15} \mathrm{~N}_{3} \mathrm{O}_{4}$ : C 61.34, $\mathrm{H}$ 4.83, N 13.41; Found: C 61.16, H 4.51, N 13.04.

Ethyl 4-oxo-8,9-dimethylfuro[3,2-e]pyrimido[1,2-c]pyrimidine-3-carboxylate (3b): m.p.: $210^{\circ} \mathrm{C}$, IR (KBr, $v_{\max }$, $\left.\mathrm{cm}^{-1}\right)$ : 1727, $1680(\mathrm{C}=\mathrm{O}), 1604,1506(\mathrm{C}=\mathrm{C}, \mathrm{C}=\mathrm{N}) .{ }^{1} \mathrm{H}$ NMR $\left(\mathrm{CDCl}_{3}\right): \delta 8.9(\mathrm{~s}, 1 \mathrm{H}, \mathrm{Ar}-\mathrm{H}$ at C6), $\delta 7.21(\mathrm{~s}, 1 \mathrm{H}, \mathrm{Ar}-\mathrm{H}$ at $\mathrm{C} 2)$, 4.35-4.37 (q, $J=6.97 \mathrm{~Hz}, 2 \mathrm{H}, \mathrm{CH}_{2}$-ester), 2.51 (s, 3H, $\mathrm{CH}_{3}$ ), $2.1\left(\mathrm{~s}, 3 \mathrm{H}, \mathrm{CH}_{3}\right), 1.46-1.48$ (t, $J=6.01 \mathrm{~Hz}, 3 \mathrm{H}, \mathrm{CH}_{3}$-ester). MS: $287\left(\mathrm{M}^{+}\right)$, Anal. calcd. (\%) for $\mathrm{C}_{14} \mathrm{H}_{13} \mathrm{~N}_{3} \mathrm{O}_{4}$ : C 58.53, H 4.56, N 14.63, Found: C 58.26, H 4.71, N 14.32.

Ethyl 4-oxo-8,9-diphenylfuro[3,2-e $]$ pyrimido[1,2-c]pyrimidine-3-carboxylate (3c): m.p.: $204^{\circ} \mathrm{C}$, IR (KBr, $v_{\max }$, $\left.\mathrm{cm}^{-1}\right): 1730,1690(\mathrm{C}=\mathrm{O}), 1610,1512(\mathrm{C}=\mathrm{C}, \mathrm{C}=\mathrm{N}) .{ }^{1} \mathrm{H}$ NMR $\left(\mathrm{CDCl}_{3}\right): \delta 8.87(\mathrm{~s}, 1 \mathrm{H}, \mathrm{Ar}-\mathrm{H}$ at C6), $\delta 7.0-7.49(\mathrm{~m}, 11 \mathrm{H}, \mathrm{Ar}-$ $\mathrm{H}), 4.35-4.36$ (q, $J=7.0 \mathrm{~Hz}, 2 \mathrm{H}, \mathrm{CH}_{2}$-ester), 1.37-1.41 (t, $J=$ $6.83 \mathrm{~Hz}, 3 \mathrm{H}, \mathrm{CH}_{3}$-ester). MS: $411\left(\mathrm{M}^{+}\right)$, Anal. calcd. (\%) for $\mathrm{C}_{24} \mathrm{H}_{17} \mathrm{~N}_{3} \mathrm{O}_{4}$ : C, 70.07; H, 4.16; N, 10.21; Found: C, 70.38; H, $4.32 ; \mathrm{N}, 10.51$.

General procedure for synthesis of 4-oxo-8,9-disubstituted furo[3,2-e]pyrimido[1,2-c]pyrimidine-3-carboxylic acid (4a-c): To a solution of compound $3(0.005 \mathrm{~mol})$ in ethanol $(15 \mathrm{~mL})$ was added $2 \mathrm{~N} \mathrm{NaOH}(5 \mathrm{~mL})$ and the reaction mixture was heated under reux for $1 \mathrm{~h}$ and allowed to cool, neutralized with dilute $\mathrm{HCl}$ solution and the obtained solid was ltered, washed with cold water, dried and crystallized.

4-Oxocyclohexa[8,9] furo[3,2-e]pyrimido[1,2-c]pyrimidine-3-carboxylic acid (4a): Yield: $53 \%$, m.p.: $230{ }^{\circ} \mathrm{C}$, IR $\left(\mathrm{KBr}, \mathrm{v}_{\max }, \mathrm{cm}^{-1}\right)$ : 3475-3258 (b,OH), 1728, $1680(\mathrm{C}=\mathrm{O})$, $1600,1505(\mathrm{C}=\mathrm{C}, \mathrm{C}=\mathrm{N}) .{ }^{1} \mathrm{H}$ NMR (DMSO- $\left.d_{6}\right): \delta 8.91(\mathrm{~s}, 1 \mathrm{H}, \mathrm{Ar}-\mathrm{H}$ at C6), $\delta 7.45$ (s, 1H, Ar-H at C2), 3.26-3.29 (t, $J=3.72 \mathrm{~Hz}, 2 \mathrm{H}$, $\left.\mathrm{CH}_{2} \mathrm{CH}_{2} \mathrm{CH}_{2} \underline{\mathrm{CH}}_{2}\right), 2.95-2.97$ (t, $J=4.55 \mathrm{~Hz}, 2 \mathrm{H}, \underline{\mathrm{CH}_{2}} \mathrm{CH}_{2} \mathrm{CH}_{2} \mathrm{CH}_{2}$ ), 1.93-1.99 (m, $J=6.55 \mathrm{~Hz}, 4 \mathrm{H}, \mathrm{CH}_{2} \mathrm{CH}_{2} \mathrm{CH}_{2} \mathrm{CH}_{2}$ ), MS: 457 $\left(\mathrm{M}^{+}\right)$, Anal. calcd. (\%) for $\mathrm{C}_{26} \mathrm{H}_{23} \mathrm{~N}_{3} \mathrm{O}_{5}: \mathrm{C}, 68.26 ; \mathrm{H}, 5.7 ; \mathrm{N}$, 9.19; Found: C, 68.16; H, 5.51; N, 9.01.

4-Oxo-8,9-dimethylfuro[3,2-e $]$ pyrimido[1,2-c $]$ pyrimidine-3-carboxylic acid (4b): Yield: 55 \%, m.p.: $234{ }^{\circ} \mathrm{C}$, IR $\left(\mathrm{KBr}, \mathrm{v}_{\max }, \mathrm{cm}^{-1}\right): 3480-3260(\mathrm{~b}, \mathrm{OH}), 3255(\mathrm{NH}), 1730,1683$ $(\mathrm{C}=\mathrm{O}), 1596,1508(\mathrm{C}=\mathrm{C}, \mathrm{C}=\mathrm{N}) .{ }^{1} \mathrm{H}$ NMR $\left(\mathrm{DMSO}-d_{6}\right): \delta 8.9$ (s, 1H, Ar-H at C6), $\delta 7.6$ (s, $1 \mathrm{H}, \mathrm{Ar}-\mathrm{H}$ at C2), 2.32 (s, 3H, $\mathrm{CH}_{3}$ ), 1.99 (s, 3H, $\mathrm{CH}_{3}$ ), MS: $259\left(\mathrm{M}^{+}\right)$, Anal. calcd. (\%) for $\mathrm{C}_{12} \mathrm{H}_{9} \mathrm{~N}_{3} \mathrm{O}_{4}$ : C, 55.60; H, 3.5; N, 16.21; Found: C, 55.36; H, 3.51; N, 16.01.

4-Oxo-8,9-diphenylfuro[3,2-e ]pyrimido[1,2-c]pyrimidine-3-carboxylic acid (4c): Yield: $50 \%$, m.p.: $224{ }^{\circ} \mathrm{C}$, IR $\left(\mathrm{KBr}, v_{\max }, \mathrm{cm}^{-1}\right): 3487-3262$ (b, OH), 1727, $1681(\mathrm{C}=\mathrm{O}), 1602$, $1508(\mathrm{C}=\mathrm{C}, \mathrm{C}=\mathrm{N}) .{ }^{1} \mathrm{H}$ NMR (DMSO-d $)$ : $\delta 9.1(\mathrm{~s}, 1 \mathrm{H}, \mathrm{Ar}-\mathrm{H}$ at C6), $\delta 8.1-7.3$ (m, 11H, Ar-H). MS: $383\left(\mathrm{M}^{+}\right)$, Anal. calcd. (\%) for $\mathrm{C}_{22} \mathrm{H}_{13} \mathrm{~N}_{3} \mathrm{O}_{4}$ : C, 68.93; H, 3.42; N, 10.96; Found: C, 68.56; $\mathrm{H}, 3.61 ; \mathrm{N}, 11.09$.

Synthesis of ethyl 2-(4-amino-5,6-diphenyl-7-hydrofuro[3,4- $d]$ pyrimidin-6-ylidene)-3-oxopropionoate (6c): To a suspension of compound $\mathbf{3 c}(0.005 \mathrm{~mol})$ in ethanol $(15 \mathrm{~mL})$, hydrazine hydrate $(1.0 \mathrm{~g}, 0.95 \mathrm{~mL}, 0.02 \mathrm{~mol})$ was added. The reaction mixture was heated under reux for $1 \mathrm{~h}$ and allowed to cool. The obtained solid was ltered, washed with cold ethanol, dried and crystallized. Yield: $55 \%$. m.p.: $271-72{ }^{\circ} \mathrm{C}$, IR (KBr, $\left.v_{\max }, \mathrm{cm}^{-1}\right)$ : 3284-3276, 3105-3101 (NH); 1693-1688 $(\mathrm{C}=\mathrm{O})$; 1614-1612 (C=C). ${ }^{1} \mathrm{H}$ NMR (DMSO- $\left.d_{6}\right)$ : 12.54 (s, $1 \mathrm{H}, \mathrm{NH}$, $\mathrm{D}_{2} \mathrm{O}$ exchangeable), 8.52 (s, $\left.1 \mathrm{H}, \mathrm{CHO}\right), \delta 7.52-7.69(\mathrm{~m}, 10 \mathrm{H}$, Ar-H), 7.27 (s, 2H, $\mathrm{NH}_{2}, \mathrm{D}_{2} \mathrm{O}$ exchangeable), $\delta$ 4.16-4.21 (q, $J=7.06 \mathrm{~Hz}, 2 \mathrm{H}, \mathrm{CH}_{2}$-ester); $\delta 1.19-1.26(\mathrm{t}, J=7.2 \mathrm{~Hz}, 3 \mathrm{H}$, $\mathrm{CH}_{3}$-ester); MS: $401\left(\mathrm{M}^{+}\right)$, Anal. calcd. (\%) for $\mathrm{C}_{23} \mathrm{H}_{19} \mathrm{~N}_{3} \mathrm{O}_{4}$ : C, 68.82; H, 4.77; N, 10.47; Found: C, 68.56; H, 4.61; N, 10.09 .

\section{RESULTS AND DISCUSSION}

The conventional method involves two steps, in the first step 5,6-disubstituted furo[2,3- $d$ ] pyrimidin-4-amines (1) were heated with EMME at $130-140{ }^{\circ} \mathrm{C}$ for $3.5-4 \mathrm{~h}$ to give diethyl $\mathrm{N}$-[5,6-substituted [2,3-d]pyrimidin-4-yl]aminomethylenemalonates (2), which on cyclization in boiling diphenyl ether at $250{ }^{\circ} \mathrm{C}$ for $1.5-2.0 \mathrm{~h}$ provided ethyl 4-oxo-8,9-disubstituted furo[3,2-e]pyrimido[1,2-c]pyrimidine-3-carboxylates $(3)$ in $52-62 \%$ overall yields from furo[2,3- $d]$ pyrimidine-4-amines 1 (Method A), while, microwave assisted cyclocondensation of 1 with EMME under solvent-free condition (Method B) gave identical compound 3 within 8-9 min in 81-85\% overall yield in a single step (Scheme-I). Thus microwave assisted one pot synthesis of furopyrimidopyrimidines (3) remarkably found advantageous over the conventional methodology because of rapid, solvent free, step reducing cleaner reaction with easy workup and increased yield. The comparison between conventional and microwave methodologies has been shown in Table-1.

TABLE-1

A COMPARISON BETWEEN CONVENTIONAL AND MICROWAVE ASSISTED SYNTHESIS OF FURO[3,2-e]PYRIMIDO[3,2-e]PYRIMIDINES (3)

\begin{tabular}{|c|c|c|c|c|}
\hline \multirow[t]{2}{*}{ Entry } & \multicolumn{2}{|c|}{$\begin{array}{c}\text { Conventional } \\
\text { Method-A }\end{array}$} & \multicolumn{2}{|c|}{$\begin{array}{c}\text { Microwave } \\
\text { Method-B }\end{array}$} \\
\hline & Time (h) & Yield (\%) & Time (min) & Yield (\%) \\
\hline $3 a$ & 5.0 & 52 & 8.0 & 81 \\
\hline $\mathbf{3 b}$ & 5.5 & 62 & 8.5 & 83 \\
\hline $3 c$ & 6.0 & 51 & 9.0 & 85 \\
\hline
\end{tabular}

The ester group in furopyrimidopyrimidines (3) has been further explored chemically for alkaline hydrolysis and hydrazinolysis. Hydrolysis of ester group with $2 \mathrm{~N}$ alcoholic sodium hydroxide under heating gave 4-oxo-8,9-disubstituted furo[3,2-e]pyrimido[1,2-c]pyrimidine-3-carboxylic acid (4), while reaction with hydrazine hydrate under reflux for $1 \mathrm{~h}$ in ethanol unexpectedly underwent ring opening same as reported by Razik et al. [20] and transformed to aminoesters 6 instead of the expected 4-oxo-9,10-disubstitutedfuro[3,2-e]pyrimido-[1,2c]pyrimidine-3-carboxylic acid hydrazide (5). IR ( $\mathrm{KBr})$ spectra of compound 3 showed band near $3258-3255 \mathrm{~cm}^{-1}$ responsible for $\mathrm{NH}$ along with two sharp characteristic bands for carbonyl group of two ester functionality at $1700-1670 \mathrm{~cm}^{-1}$. Absorption bands due to $\mathrm{C}=\mathrm{C}$ and $\mathrm{C}=\mathrm{N}$ vibrations were found in the region $1603-1500 \mathrm{~cm}^{-1}$. The absence of amino vibrations in the region $3500-3400 \mathrm{~cm}^{-1}$ supported the formation of uncyclized inter- 
mediate 2. ${ }^{1} \mathrm{H} \mathrm{NMR}\left(\mathrm{CDCl}_{3}\right)$ of $\mathbf{2}$ exhibited a deuterium exchangeable doublet at $\delta 10.4-11.63$ integrating for $1 \mathrm{H}$ for $\mathrm{NH}$ proton, a doublet due to vinyl proton in the region $\delta 9.22-9.36$ and singlet at $\delta 8.63-8.78$ due to pyrimidine ring proton. Twin quartet and triplet in the region $\delta 4.02-4.39$ and $\delta 1.22-1.42$ each integrating for $3 \mathrm{H}$ and $2 \mathrm{H}$ respectively were responsible for two ethyl groups present in malonate functionality of 2 . In IR $(\mathrm{KBr})$ spectra of 3 the absence of band near $3256-3250 \mathrm{~cm}^{-1}$ due to NH vibrations suggested the formation of angular furopyrimidopyrimidines (3). The carbonyl group of ester exhibited band in the area $1730-1720 \mathrm{~cm}^{-1}$ whereas, absorption due to lactone was found to be shifted $20-30 \mathrm{~cm}^{-1}$ higher wave number as compared to ketones of uncyclized malonates producing a sharp band in the area $1690-1680 \mathrm{~cm}^{-1}$. Pyrimidine protons at C6 and C2 appeared at $\delta 8.87-9.1$ and $\delta 7.21-7.25$ as singlet each integrating for one proton, whereas ethyl protons of ester group appeared as a triplet at $\delta 1.37-1.48$ integrating for $3 \mathrm{H}$ and a quartet at $\delta 4.35-4.46$ integrating for $2 \mathrm{H}$ in the ${ }^{1} \mathrm{H} \mathrm{NMR}\left(\mathrm{CDCl}_{3}\right)$ spectra of compound $\mathbf{3}$. IR ( $\mathrm{KBr}$ ) spectra of compound $\mathbf{4}$ showed a broad band in the range $3500-3260 \mathrm{~cm}^{-1}$ due to for hydroxyl group of acid along with two bands for acid carbonyl and cyclic ketone at $1728-1680 \mathrm{~cm}^{-1}$. The absence of triplet and quartet in the ${ }^{1} \mathrm{H}$ NMR (DMSO- $d_{6}$ ) spectra of compound 4 and the presence of singlet for $\mathrm{C} 6$ at 9.1-8.91 and $\mathrm{C} 2$ at 7.45-7.6 suggested the acid formation. IR ( $\mathrm{KBr}$ ) displayed the bands in the area 3500-3400 and 3200 for $\mathrm{NH}, 1740 \mathrm{~cm}^{-1}$ for aldehyde carbonyl and $1720 \mathrm{~cm}^{-1}$ for ester carbonyl groups. Investigation of ${ }^{1} \mathrm{H}$ NMR (DMSO- $d_{6}$ ) spectrum of compound $\mathbf{6 c}$ revealed two deuterium exchangeable signals due to $\mathrm{NH}$ and $\mathrm{NH}_{2}$ moieties in addition to a triplet and a quartet corresponding to ethyl ester fragment. Other protons were located at their expected chemical shifts. The mass spectrum of ethyl 4-oxo-8,9-diphenyl-furo[3,2-e]pyrimido[1,2-c]pyrimidine-3-carboxylate (3c) exhibited a characteristic molecular ion peak at $m / e$ 411. The fragment ion $\left(\mathrm{M}-\mathrm{COOC}_{2} \mathrm{H}_{5}\right)$ was obtained at $m / e=338$ (Scheme-III).

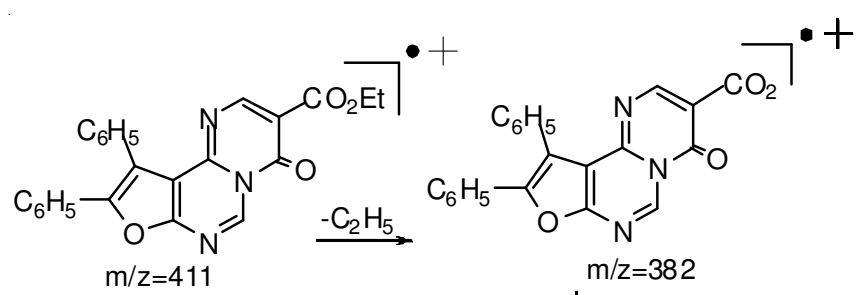<smiles>CC(C)C(C)C[C@H](C)I</smiles>

$\mathrm{m} / \mathrm{z}=310$

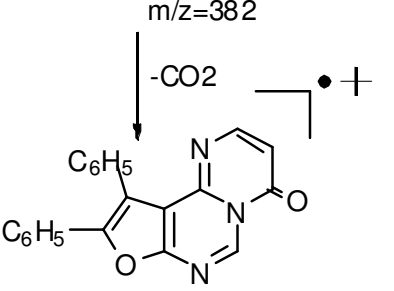

$\mathrm{m} / \mathrm{z}=338$

\section{Conclusion}

Scheme-III

An efficient, simple, step reducing, fast, solvent-free and high yielding one pot synthetic route for newly synthesized furopyrimidopyrimidines by microwave acceleration is described. Alkaline hydrolysis for acid formation and treatment with hot hydrazine hydrate to ring opening forming aminoester have been reported.

\section{ACKNOWLEDGEMENTS}

The authors thank the Regional Sophisticated Instrumentation Center, Central Drug Research Institute, Lucknow, India and National Facility for Drug Discovery Centre, Rajkot, India for ${ }^{1} \mathrm{H}$ NMR and mass spectral analysis and M.G. Science Institute, Ahmedabad, India for providing the facilities to carry out this work.

\section{REFERENCES}

1. A. Loupy, A. Petit, J. Hamelin, F. Texier-Boullet, P. Jacquault and D. Mathé, Synthesis, 1213 (1998);

https://doi.org/10.1055/s-1998-6083.

2. R.S. Varma, Pure Appl. Chem., 73, 193 (2001); https://doi.org/10.1351/pac200173010193.

3. I. Oussaid, N. Thach and A. Loupy, Tetrahedron Lett., 38, 2451 (1997); https://doi.org/10.1016/S0040-4039(97)00366-3.

4. R.G. Gould Jr. and W.A. Jacobs, J. Am. Chem. Soc., 61, 2890 (1939); https://doi.org/10.1021/ja01265a088.

5. L.A. Paquette, D. Crich and P.L. Fuchs, Encyclopedia of Reagents for Organic Synthesis 3, John Wiley \& Sons, New York, p. 1816 (1995).

6. J. Sanddstrome, Top. Stereochem., 83, 14 (1983).

7. M. Ihara, K. Noguchi, T. Ohsawa, K. Fukumoto and T. Kametani, J. Org. Chem., 48, 3150 (1983); https://doi.org/10.1021/jo00167a003.

8. S. Hibino, E. Sugino, T. Kuwada, N. Ogura, K. Sato and T. Choshi, J. Org. Chem., 57, 5917 (1992);

https://doi.org/10.1021/jo00048a026.

9. D.L. Boger and C.E. Brotherton, J. Am. Chem. Soc., 108, 6695 (1986); https://doi.org/10.1021/ja00281a041.

10. A. Kaczor and D. Matosiuk, Curr. Org. Chem., 9, 1237 (2005); https://doi.org/10.2174/1385272054863943.

11. N. Katagiri, H. Akatsuka, T. Haneda, C. Kaneko and A. Sera, J. Org. Chem., 53, 5464 (1988); https://doi.org/10.1021/jo00258a013.

12. C.G. Dave and M.C. Shukla, J. Heterocycl. Chem., 34, 1805 (1997); https://doi.org/10.1002/jhet.5570340627.

13. C.G. Dave and R.D. Shah, Heterocycles, 51, 1819 (1999); https://doi.org/10.3987/COM-99-8545.

14. N.D. Desai, J. Heterocycl. Chem., 43, 1343 (2006); https://doi.org/10.1002/jhet.5570430530.

15. S. Caddick, Tetrahedron, 51, 10403 (1995); https://doi.org/10.1016/0040-4020(95)00662-R.

16. P. Lidström, J. Tierney, B. Wathey and J. Westman, Tetrahedron, 57, 9225 (2001); https://doi.org/10.1016/S0040-4020(01)00906-1.

17. M. Nüchter, U. Müller, B. Ondruschka, A. Tied and W. Lautenschläger, Chem. Eng. Technol., 26, 1207 (2003); https://doi.org/10.1002/ceat.200301836.

18. C.O. Kappe, Angew. Chem. Int. Ed., 43, 6250 (2004); https://doi.org/10.1002/anie.200400655.

19. K. Tanaka and F. Toda, Chem. Rev., 100, 1025 (2000); https://doi.org/10.1021/cr940089p.

20. H.A. Razik and A.E. Abdel Wahab, Arch. Pharm. Chem. Life Sci., 11, 184 (2011).

21. C.G. Dave, P.R. Shah and S.P. Upadhyaya, J. Indian Chem. Soc., 64, 713 (1987).

22. C.G. Dave, A.B. Shah and H.C. Shah, J. Heterocycl. Chem., 34, 937 (1997); https://doi.org/10.1002/jhet.5570340335.

23. C.G. Dave and R.D. Shah, J. Heterocycl. Chem., 35, 1295 (1998); https://doi.org/10.1002/jhet.5570350609.

24. C.G. Dave and N.D. Desai, J. Heterocycl. Chem., 36, 729 (1999); https://doi.org/10.1002/jhet.5570360325.

25. C.G. Dave and R.D. Shah, J. Heterocycl. Chem., 37, 757 (2000); https://doi.org/10.1002/jhet.5570370415.

26. C.G. Dave and R.D. Shah, Molecules, 7, 554 (2002); https://doi.org/10.3390/70700554.

27. N.D. Desai and R.D. Shah, Synthesis, 3275 (2006); https://doi.org/10.1055/s-2006-950206.

28. R.D. Shah, Chemistry for Sustain. Dev., 19, 321 (2011). 
29. R.D. Shah, N.M. Shah and V. Ramani, Int. J. Sci. Res., 5, 207 (2016).

30. R.D. Shah, M.M. Jotani and E.R.T. Tiekink, ActaCryst., E66, o3305 (2010);

https://doi.org/10.1107/S1600536810048373.

31. R.D. Shah, M.M. Jotani and J.P. Jasinski, ActaCryst., E66, o601 (2010); https://doi.org/10.1107/S160053681000485X.

32. R.D. Shah, M.M. Jotani and J.P. Jasinski, ActaCryst, E66, o212 (2010); https://doi.org/10.1107/S1600536809053653.
33. M.M. Jotani, R.D. Shah, J.P. Jasinski and R.J. Butcher, ActaCryst., E66, 0574 (2010); https://doi.org/10.1107/S1600536810004368.

34. M.M. Jotani, R.D. Shah and E.R.T. Tiekink, ActaCryst., E66, o805 (2010);

https://doi.org/10.1107/S160053681000869X

35. K. Gewald, Chem. Ber, 99, 1002 (1966); https://doi.org/10.1002/cber.19660990340. 